\title{
Evaluation of Small Mass Spectrometer Systems for Permanent Gas Analysis
}

\author{
C Richard Arkin and Timothy P. Griffin \\ Dynacs Inc., DNX-14, Kennedy Space Center, FL, USA \\ Andrew K. Ottens \\ Department of Chemistry, University of Florida, Gainesville, FL, USA
}

Jorge A. Diaz

Universidad de Costa Rica, Escuela de Física, San José, Costa Rica

\author{
Duke W. Follistein, Fredrick W. Adams, and William R. Helms
}

NASA, YA-D2-E2, Kennedy Space Center, FL, USA

\begin{abstract}
This work is aimed at understanding the aspects of designing a miniature mass spectrometer (MS) system. Several types of small MS systems are evaluated and discussed, including linear quadrupole, quadrupole ion trap, time of flight, and sector. Analysis of hydrogen, helium, oxygen, and argon in a nitrogen background with the concentrations of the components of interest ranging from 0 to 5000 parts per million (ppm). The performance of each system in terms of accuracy, precision, limits of detection, response time, recovery time, scan rate, size, and weight is assessed. The relative accuracies of the systems varied from $<1 \%$ to $\sim 40 \%$ with an average below $10 \%$. Relative precisions varied from $1 \%$ to $20 \%$, with an average below $5 \%$. The detection limits had a large distribution, ranging from 0.2 to $170 \mathrm{ppm}$. The systems had a diverse response time ranging from 4 to $210 \mathrm{~s}$, as did the recovery time with a 6-to-210-s distribution. Most instruments had scan times near $1 \mathrm{~s}$; however, one instrument exceeded $13 \mathrm{~s}$. System weights varied from 9 to $52 \mathrm{~kg}$ and sizes ranged from $15 \times 10^{3} \mathrm{~cm}^{3}$ to $110 \times 10^{3}$ $\mathrm{cm}^{3}$. A performance scale is set up to rank each system, and an overall performance score is given to each system. (J Am Soc Mass Spectrom 2002, 13, 1004-1012) (C) 2002 American Society for Mass Spectrometry
\end{abstract}

$\mathrm{T}$ There is an ever-increasing need for smaller mass spectrometer (MS) systems. A multitude of commercial and government sectors, such as the military, environmental agencies, and industrial manufacturers of semiconductors, refrigerants, and petroleum products, would find a small, portable, rugged, and reliable MS system beneficial. Compared to current commercial systems, small MS systems are generally more easily transported, less expensive, operated in more diverse environments, and enable real-time analysis. Such systems have many potential applications such as environmental analysis, process monitoring, leak detection, and hazardous chemical detection. At Kennedy Space Center (KSC) there is a need for small, point-sensor MS systems for a variety of tasks. Such tasks include the monitoring of hazardous gases around the Space Shuttle prior to launch, the detection of toxic

Published online June 27, 2002

Address reprint requests to C Richard Arkin, Dynacs Inc., DNX-14, P.O. Box 21087, John F. Kennedy Space Center, FL 32815-0087, USA. E-mail: Richard. Arkin-1@ksc.nasa.gov propellant vapors such as hydrazine derivatives, and process control of a fuel production plant on Mars.

Mass spectrometer systems have been used at KSC since the beginning of the space program [1]. The primary reasons mass spectrometers are used are their excellent limits of detection (LOD), response time, recovery rate, accuracy, and capability to monitor and differentiate several species. One aspect of the work at $\mathrm{KSC}$ is to develop systems that monitor the cryogenic fuels, liquid hydrogen, and liquid oxygen used for launching the Space Shuttle. A buildup of gaseous hydrogen or oxygen during fueling or launch creates a hazardous environment. For this reason, areas of potential hazard are purged with nitrogen and analyzed for hydrogen, helium, oxygen, and argon. (Hydrogen and oxygen indicate a leak of the cryogens, argon indicates an air leak, and helium is used for leak checking prior to fueling.)

Currently, a large mass spectrometer system $[2,3]$ performs this task, using long transport lines to draw in samples from various points around the Shuttle. This arrangement is depicted in Figure 1. The current system 


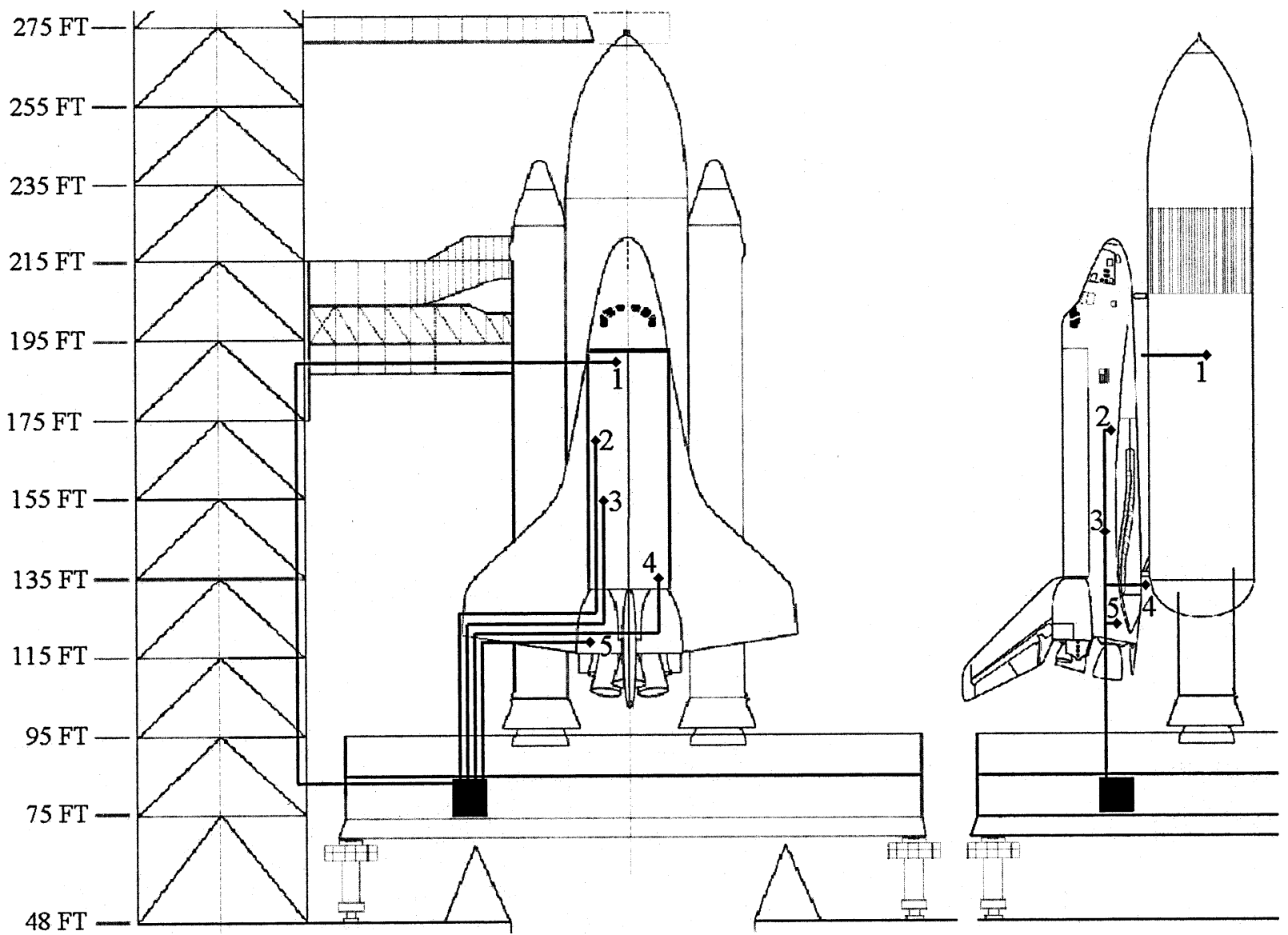

Figure 1. Location of current instrument and sample positions. The current gas detection system, HGDS 2000, is located in the Mobile Launcher Platform (MLP). As a result, the samples must be transported from around the launch vehicle to the detection system. The HGDS 2000 system is depicted by the black square with the 5 sample lines attached. The approximate positions of the 5 sample locations are shown and are the (1) external tank-internal tank, $387 \mathrm{ft}$, (2) midbody, $227 \mathrm{ft}$, (3) payload bay, $226 \mathrm{ft}$, (4) hydrogen side umbilical, $209 \mathrm{ft}$, and (5) aft, $200 \mathrm{ft}$.

has several undesirable attributes. Since the sampling points are removed from the MS system, long transport lines (up to approximately $115 \mathrm{~m}[370 \mathrm{ft}]$ ) are used. As a result of the distance and flow rate, the sample being analyzed is actually 15 to $30 \mathrm{~s}$ old. Among the various problems that this delay in response poses, one of the most serious is that monitoring for leaks during the last fraction of a minute prior to launch is precluded. Sequential, round-robin sampling of the lines causes additional delays. This system is also very large $\left(\sim 3.65 \times 10^{6}\right.$ $\left.\mathrm{cm}^{3}\right)$, heavy $(\sim 770 \mathrm{~kg}[1700 \mathrm{lbs}])$, and expensive $(\sim \$ 1 \mathrm{M})$. Last, if more sampling points were added, more transport lines would be needed, thus adding to the size and weight of the system as well as creating even longer delays between consecutive readings due to the roundrobin scanning method.

There is great interest in replacing this stationary system with several miniature $[4,5]$, portable, rugged mass spectrometers to act as point sensors that can be placed at the sampling locations. A small, lightweight system would provide several advantages. First, because it is a point sensor, there is no need for long transport lines, thus eliminating the delay between sample uptake and analysis. Second, with multiple sensors, several locations can be monitored simultaneously. Additional sampling points would not create a delay between consecutive scans. Third, small instruments tend to cost less than their larger counterparts. Fourth, in the event one system fails, several entire systems can be on the shelf available for installation as needed. Currently, when an instrument fails, that instrument is evaluated and parts are repaired or replaced. As a result, numerous parts must be stocked, qualified personnel must spend valuable time involved in repairs and the potential exists for a costly delay in launch. Also, with the systems being lightweight and portable, there is the potential that these systems can remain functional during the launch, ascent, orbit, and descent.

In order to achieve this goal, a miniature MS system should have the following attributes. The system should be small $\left(<35,000 \mathrm{~cm}^{3}\right)$, lightweight $(<10 \mathrm{~kg})$, power-efficient $(<250 \mathrm{~W})$, rugged (survive $18 \mathrm{~g}$ 's), and relatively inexpensive $(<\$ 20,000)$. The system should also have low limits of detection $(<10 \mathrm{ppm})$, fast response and recovery times $(<30 \mathrm{~s})$, rapid scan times 
Table 1. Mass spectrometer systems evaluated

\begin{tabular}{lll}
\hline System Type & \multicolumn{1}{c}{ Manufacturer/Model } & Analyzer Type \\
\hline \hline SBIR & Ferran & Linear Quadrupole Array \\
& IonWerks (TOF) & o-TOF \\
COTS & Stanford Research Systems RGA-100 (SRS) & Linear Quadrupole \\
& Linear Quadrupole \\
& Monitor Instruments MG-2100 & Cycloidal Focus \\
In-House & Thermo Finnigan Polaris-Q & Quadrupole Ion Trap \\
& University of Florida/KSC (UF-IT) & Quadrupole Ion Trap \\
& University of Minnesota/KSC (CDFMS) & Double Focus Sector \\
\hline
\end{tabular}

$(<1 \mathrm{~s})$, and provide accurate results ( $<10 \%$ error) over a sustained period of time ( $\sim 12$ hours).

Eight small mass spectrometer systems were evaluated for this application. Hydrogen, helium, oxygen, and argon were measured at low levels (100 to 5000 ppm) in a nitrogen background. Under these conditions, the various figures of merit were determined.

\section{Experimental}

The eight instruments evaluated can be organized into three categories: systems involved in the Small Business Innovative Research (SBIR) grant program, commercial off-the-shelf (COTS) instruments, and systems developed in-house. Table 1 summarizes the instruments, models, and analyzer types of the systems evaluated. Note that the Ferran (San Diego, CA), IonWerks (ORTOF, Houston, TX), MG-2100 (Monitor Instruments Company, Cheswick, PA), and Polaris-Q (Thermo Finnigan, San Jose, CA) are complete systems. The SRS and XPR-2 (Inficon, East Syracuse, NY) are only analyzers; the rest of the systems were built in-house.

The Ferran [6-9] system utilizes a miniature linear quadrupole array consisting of 16 rods. Each rod has a diameter of $1 \mathrm{~mm}$ and a length of $10 \mathrm{~mm}$. The operating frequency for this system is $16 \mathrm{MHz}$, the operating pressure is $9 \times 10^{-4}$ torr, and a Faraday cup is used for detection. The IonWerks Time-of-Flight (TOF) is an orthogonally accelerated reflectron. A unique anode detector is used, which is claimed to increase the dynamic range [10]. The Stanford Research Systems RGA-100 (Sunnyvale, CA) is a linear quadrupole with rod diameters of 0.25 in and length of 4.5 in. The operating frequency is $2.7648 \mathrm{MHz}$, and both a Faraday cup and electron multiplier detectors are available, although the multiplier was used here. For this application, the system operates best when the analyzer is in the mid- $10^{-5}$-torr pressure range. The Inficon XPR-2 is a linear quadrupole with hyperbolic rods with an inscribed radius of 0.013 in and length of $0.5 \mathrm{in}$. The XPR-2 operates at $13 \mathrm{MHz}$ and has both a Faraday cup and channel electron multiplier detectors. This analyzer generally operates with the electron multiplier and an analyzer pressure in the low $10^{-4}$-torr range. The Monitor Instruments MG-2100 is a cycloidal focusing sector. The Thermo Finnigan Polaris-Q is a quadrupole ion trap of stretched geometry with an internal ring radius of $7 \mathrm{~mm}$, operating at a frequency of $1.03 \mathrm{MHz}$. Due to the geometry and operating frequency, the Polaris- $Q$ is unable to monitor hydrogen and helium. A second quadrupole ion trap, the UF-IT, was developed at the University of Florida [11, 12]. This ion trap is of stretched geometry with an internal ring radius of 10 $\mathrm{mm}$ and operates at a frequency of $2.5 \mathrm{MHz}$. The compact double-focusing sector mass spectrometer (CDFMS) is a crossed electric and magnetic field sector analyzer with a $90^{\circ}$ geometry that was developed at the University of Minnesota [13-16]. The magnetic field strength was $0.75 \mathrm{~T}$, and the sector radius was $2 \mathrm{~cm}$.

With the exceptions of the UF-IT, Polaris-Q, and TOF, all instruments used an Alcatel ATH 30+ turbomolecular-drag high-vacuum pump. This pump was chosen due to its high compression ratios for hydrogen and helium, as well as nitrogen $\left(10^{5}, 10^{7}\right.$, and $10^{11}$, respectively). The high compression ratios are needed for good response and recovery times between samples. The TOF has a Varian (Walnut Creek, CA) V70LP high-vacuum turbomolecular pump; the UF-IT uses a Pfeiffer model TPH-65 turbomolecular-drag pump; and the Polaris-Q uses an Edwards High Vacuum International (Asslar, Germany) model $70 \mathrm{H}$ turbomoleculardrag pump. In order to maintain clean vacuum chambers and mass analyzers, either scroll pumps or diaphragm pumps were used for backing the turbomolecular pumps and providing sample delivery. In general, a Varian 300 scroll pump was used for sample delivery, and typically a diaphragm pump was used with the turbo pumps. All systems used electron impact (EI) ionization with thoriated iridium filaments operating at $70 \mathrm{eV}$.

A similar experiment was used to evaluate all mass spectrometers. The experimental sequence is depicted in Figure 2. A zero bottle $\left(>99.995 \% \mathrm{~N}_{2}\right)$, test bottle (500 ppm $\mathrm{H}_{2}, 500$ ppm He, 500 ppm $\mathrm{O}_{2}, 100$ ppm Ar, and balance $\mathrm{N}_{2}$, nominally), and span bottle (5000 ppm $\mathrm{H}_{2}$, 5000 ppm He, 5000 ppm O, 1000 ppm Ar, and balance $\mathrm{N}_{2}$, nominally) were analyzed sequentially for approximately 5 min each. This cycle constitutes the calibration cycle, where the zero and span serve as a two-point calibration curve and the test was used to determine the quality of the calibration. All data values were determined using a 10-point data set. Following the calibration cycle, the test bottle was analyzed for $5 \mathrm{~min}$ and then returned to the zero bottle. At this time, the 


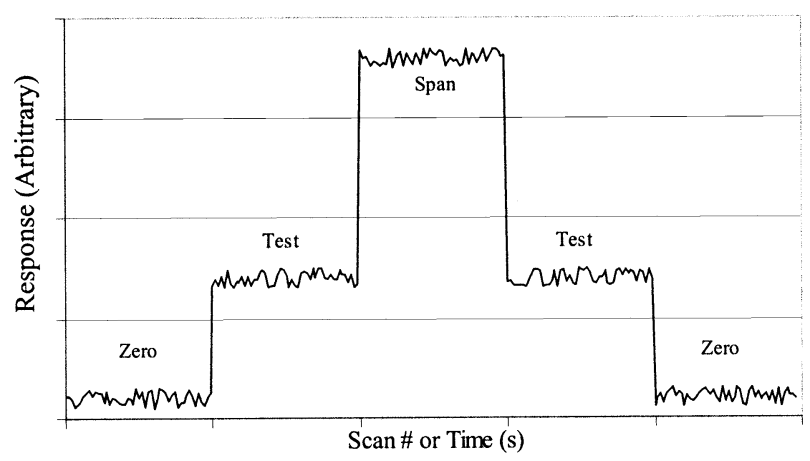

Figure 2. Example of typical evaluation experiment. The general experiment used to evaluate the mass spectrometers begins with a calibration cycle. This cycle uses a zero bottle $\left(>99.995 \% \mathrm{~N}_{2}\right)$, test bottle (500 ppm $\mathrm{H}_{2}, 500 \mathrm{ppm} \mathrm{He}, 500$ ppm $\mathrm{O}_{2}, 100 \mathrm{ppm} \mathrm{Ar}$, and balance $\mathrm{N}_{2}$, nominally), and span bottle ( $5000 \mathrm{ppm} \mathrm{H}_{2}, 5000 \mathrm{ppm}$ $\mathrm{He}, 5000$ ppm $\mathrm{O}_{2}, 1000 \mathrm{ppm} \mathrm{Ar}$, and balance $\mathrm{N}_{2}$, nominally), where each is analyzed for 5 min sequentially. The zero and span serve as a two-point calibration curve, and the test is used to determine the quality of the calibration. Following the calibration cycle, the test bottle is analyzed for $5 \mathrm{~min}$ and then returned to the zero bottle. This allows the other test parameters to be determined as described in the text.

calibration cycle was used to scale all data in terms of concentration with which the parameters of evaluation are determined.

For each scan, the SRS conducted a mini-scan of \pm 0.3 $\mathrm{Th}(\mathrm{Th} \equiv \mathrm{amu} / \mathrm{e})$ about the chosen ion to monitor. The intensity of the local maximum was the response for that ion. For both the XPR-2 and Ferran, a mini-scan of \pm 0.5 Th about the chosen monitored ion was performed with the peak intensity considered the response. Using Xcalibur (v1.2) software, the Polaris-Q ion trap was scanned from 31 to $41 \mathrm{Th}$, and the peak height was determined from the local maximum within a window of \pm 0.5 Th of the monitored ions. Each scan consisted of 10 averaged spectra, which were subjected to a twopoint boxcar average. Each UF-IT spectrum involved two sequences. The first sequence involved the analysis of hydrogen and helium, where a spectrum from 1 to 5 Th was produced. The second monitored oxygen and argon by producing a spectrum from 31 to 41 Th. The acquisition hardware recorded the maximum ion current within a window of \pm 0.5 Th of the target mass. For each scan, the above process was repeated 120 times and averaged and then subjected to a 3-point boxcar average. The TOF was operated at a sampling rate of 20 $\mathrm{kHz}$. Each spectrum consisted of an average of 20,000 samples. After the software identified the ion peak, integration of twice the full width half maximum (FWHM) on both sides of the ion peak was considered the response for that ion. For each scan of the MG-2100, a mini-scan of \pm 0.2 Th about the chosen ion to monitor was performed and the intensity of the local maximum was the response for that ion. For the CDFMS the data was acquired using a DATAQ datalogger that only recorded one intensity value for each ion collected. No local maximum was considered. For all instruments, the data was saved in a comma-delimited format as a function of scan-number and elapsed time. Using inhouse software or a standard spreadsheet program, the calibration and other data described below was calculated.

With the various definitions available for the parameters used in this study, each parameter is defined below. Accuracy, eq 1, is the ratio of the difference between the measured test concentration ([test $]_{\text {meas }}$ ) and the true concentration $\left([\text { test }]_{\text {true }}\right.$ ) to the true concentration. When shown graphically, accuracy (A) is reported as an absolute value.

$$
A=\frac{[\text { test }]_{\text {meas }}-[\text { test }]_{\text {true }}}{[\text { test }]_{\text {true }}} \times 100 \%
$$

As described in eq 2, precision (also known as relative standard deviation, RSD) is the ratio of the standard deviation $\left(s_{\text {meas }}\right.$ ) of the measured data set for the test bottle to the measured concentration of the test bottle.

$$
P=\frac{s_{\text {meas }}}{[\text { test }]_{\text {meas }}} \times 100 \%
$$

The theoretical limit of detection (LOD), eq 3, was defined as 3 times the standard deviation of the measured data set for the zero bottle.

$$
L O D=3 s_{\text {zero }}
$$

Response time was defined as the time between the bottle change from zero gas to test gas until the reading is within $95 \%$ of the average measured test value. The recovery time was defined as the elapsed time between changing the bottle from the test gas to the zero gas and a signal reading $5 \%$ of the test reading $(95 \%$ reduction in signal). Scan time was defined as the time required for one scan to be acquired and the data to be transferred. It was determined by measuring the time necessary to acquire a known number of scans and dividing by said number of scans. The system volume was determined by measuring the entire system volume including the mass analyzer, its associated electronics, vacuum system, high-vacuum pump, and rough pump. Also measured are any ion gauge controllers or other required equipment. Computers were not included. System weight was determined by weighing each individual component-again computers were not included. These evaluation parameters, along with the defined requirements, are summarized in Table 2.

\section{Results and Discussion}

Although an accuracy requirement of $10 \%$ may seem trivial for many analytical techniques, it is a realistic goal for this application considering the concentration range (ppm to the sub-percent levels) and the sample 
Table 2. Summary of experimental parameters [17]

\begin{tabular}{|c|c|c|}
\hline Parameters & Definition & Shuttle Requirements \\
\hline Accuracy & $\frac{[\text { Test }]_{\text {meas }}-[\text { Test }]_{\text {true }}}{[\text { Test }]_{\text {true }}} \times 100 \%$ & $<10 \%$ or $5 \mathrm{ppm}$, whichever is greater \\
\hline Precision & $\frac{S_{\text {Test }, \text { meas }}}{[\text { Test }]_{\text {meas }}} \times 100 \%$ & $<5 \%$ or $3 \mathrm{ppm}$, whichever is greater \\
\hline Limit of Detection & $3 s_{\text {zero }}{ }^{\mathrm{a}}$ & $\mathrm{H}_{2}, \mathrm{O}_{2}: 25$ ppm; He: 100 ppm; Ar: 10 ppm \\
\hline Response Time & $\begin{array}{l}\text { Time required for response from valve change to } \\
95 \% \text { new reading }\end{array}$ & $10 \mathrm{~s}$ \\
\hline Recovery Time & $\begin{array}{l}\text { Time after valve change to reach } 5 \% \text { of previous } \\
\text { sample reading (new sample is zero) }\end{array}$ & $30 \mathrm{~s}$ \\
\hline Scan Time & Experiment Time/Scans & $1 \mathrm{~s}$ \\
\hline Maximum Size & Sum of Individual Components & $3.5 \times 10^{4} \mathrm{~cm}^{3}$ \\
\hline Maximum Weight & Sum of Individual Components & $10 \mathrm{~kg}$ \\
\hline
\end{tabular}

aTheoretical limit of detection

${ }^{\mathrm{b}}$ Measured limit of detection

type (permanent gases). Figure 3 shows the results of the accuracy tests for each system and component evaluated. Half of the systems (SRS, XPR-2, UF-IT, and CDFMS) met the required $(<10 \%)$ accuracy. Of the remaining systems, the Polaris-Q slightly exceeded the requirements for argon with an accuracy of $13 \%$. The accuracies of the Ferran, TOF, and MG-2100 were greater than $10 \%$ for many of the analyzed components; this is considered unacceptable.

It is important to recognize that there are no clear trends with each component in regard to accuracy. For example, hydrogen is very difficult to analyze using a linear quadrupole because of the zero-blast and the very shallow pseudo-potential well [18]. In the case of the SRS, hydrogen has the best accuracy of all of the analyzed species; yet, in the case of the XPR-2, it is nearly the worst.

Figure 4 displays the precision results for the various systems and for each component analyzed. Half of the systems (SRS, Polaris-Q, UF-IT, and CDFMS) met the requirement of $<5 \%$, with the TOF slightly exceeding. The MG-2100 mildly exceeded the requirements, while the XPR-2 and Ferran had significant precision problems, with precisions exceeding 15\%. The SRS,

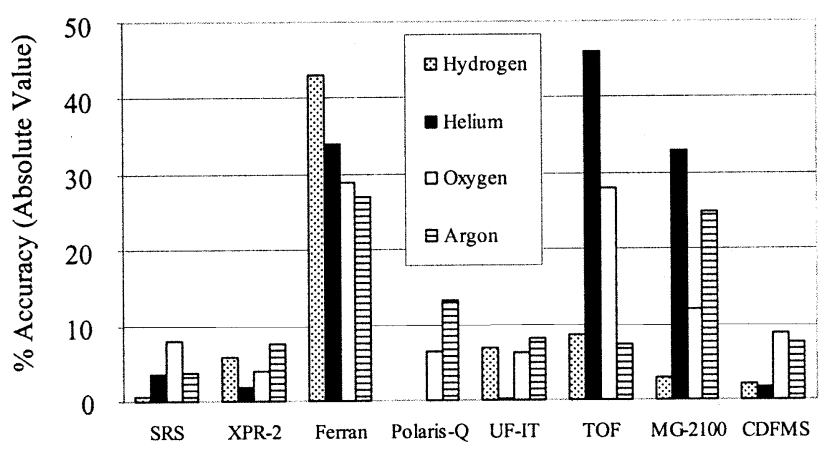

Figure 3. Comparison of the Accuracy of Each Instrument for Each Species. The accuracy is determined by comparing the true concentration of the test gas to that measured and reported in terms of absolute values. At these concentration levels, an error of $10 \%$ or less is acceptable.
Polaris-Q, and UF-IT systems were substantially better than the required 5\% precision level.

It is not clear which factors affect precision, but there are several likely sources. For example, it is believed that maintaining a stable pressure at the sample inlet is of primary importance. All of the systems evaluated here maintained a stable pressure via an in-house sample delivery system (SDS). The sample delivered to the MS is drawn downstream of a manual needle valve (to maintain a desired flow rate) and upstream of the pressure transducer and flow controller. The pressure transducer and flow controller are used in combination to control the upstream pressure. We have found that using this configuration, controlling the pressure in an upstream (rather than downstream) manner, provides faster response to changes in sample concentration. It is believed that the method by which the sample is introduced into the MS can affect the precision. Three techniques are used here. The SRS, XPR-2, Polaris-Q, and UF-IT use orifices for sample introduction. The Ferran, TOF, and MG-2100 use capillaries, and the CDFMS uses a frit for sample introduction. More often than not, the orifice technique provides better results. Also, the pressure within the analyzer may affect the

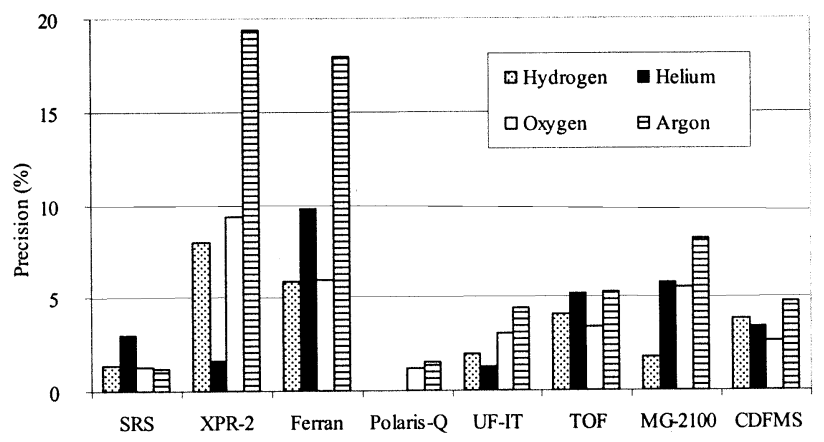

Figure 4. Comparison of the precision of each instrument for each species. The precision is the ratio of the standard deviation of the test gas measurement to the average of the test gas measurement. At these concentration levels, a $5 \%$ precision level or less is acceptable. 


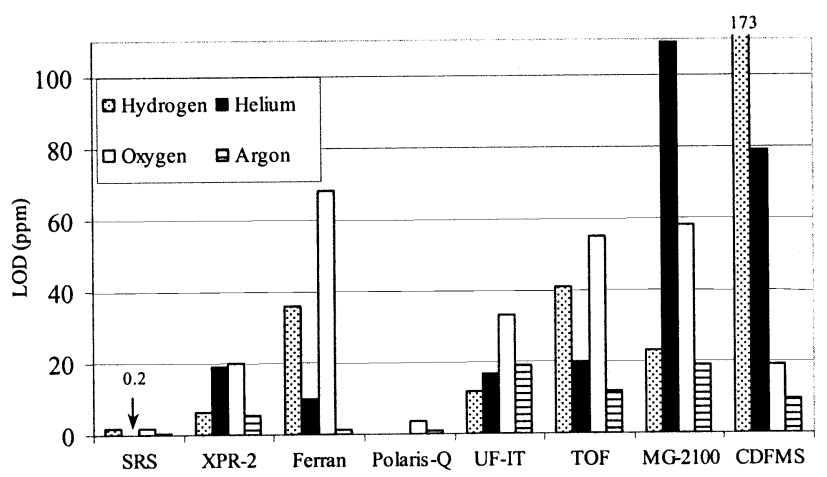

Figure 5. Comparison of the theoretical limits of detection (LOD) of each instrument for each species. The theoretical LOD is calculated to be three times the standard deviation of the zero gas measurement. The required, measured LODs are given in Table 2.

precision. The SRS, UF-IT, TOF, MG-2100, and CDFMS all operate in the mid-10 $10^{-5}$-torr range. The XPR-2 and Ferran operate in the low- $10^{-4}$-torr range, and the Polaris-Q operates in the upper $10^{-4}$ - (and lower $10^{-3}$ ) torr range. No clear trend has been observed that indicates which operating range is best for precision purposes, yet each system has a very specific pressure range in which optimum performance is found.

The limits of detection (LOD) for each instrument are shown in Figure 5. The best instrument for LOD was the SRS, while the XPR-2 also performed within specifications. With regard to detecting oxygen and argon, the Polaris-Q performed very well, but its mass range prevented it from detecting hydrogen and helium. With the exception of oxygen, the UF-IT performed quite well in this category. The Ferran performed well for argon but needs significant improvement in detection of hydrogen and helium. Although the Ferran appeared to perform well for helium, this was an artifact of the LOD calculation. The data analysis software of the Ferran imposes an ion current threshold. An ion current lower than the threshold is assigned a value of zero. As a result, the analysis of helium in the zero gas results in a data set with mostly zeros, thus artificially lowering the LOD. The TOF, MG-2100, and the CDFMS need significant improvement for all of the gases.

The LOD is one of the more challenging specifications to meet or exceed. There are a variety of reasons for this. An important factor in analyzing these gases is their high ionization potentials $\left(\mathrm{H}_{2}: 15.4 \mathrm{eV}\right.$, He: $24.6 \mathrm{eV}$, $\mathrm{N}_{2}: 15.6 \mathrm{eV}, \mathrm{O}_{2}: 12.1 \mathrm{eV}$, Ar: $15.8 \mathrm{eV}$ ). As a result, the ionization efficiency tends to be low relative to typical volatile organic compounds (VOCs). Preconcentration of the analyte through GC, as is commonly done with VOCs to achieve parts-per-billion (ppb) LODs, is not viable here due to time-per-scan considerations. Preconcentration via removal of the "carrier gas," nitrogen in this case, is another technique to improve LOD. Achieved using a gas jet separator, this works quite well for components of mass greater than the carrier gas, since jet separators act as high-pass (passing masses higher than the carrier) filters. Unfortunately, this

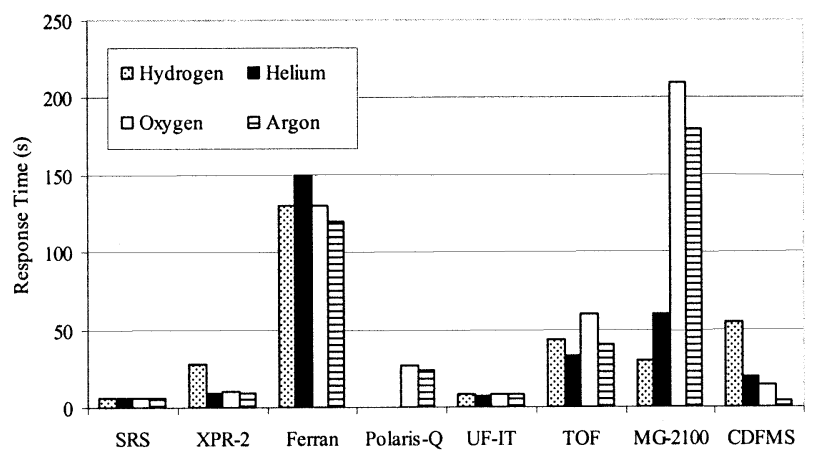

Figure 6. Comparison of the response times of each instrument for each species. Response time is measured as the time between when the zero-to-test bottle change occurs until the measured concentration is within $95 \%$ of the average measured concentration of the test gas. An acceptable response time is $10 \mathrm{~s}$ or less.

would significantly hinder hydrogen and helium analysis.

Response times for each system (and respective components) are shown in Figure 6. With a specification of $10 \mathrm{~s}$, the SRS and UF-IT met the requirements. Only hydrogen exceeded the 10-s mark for the XPR-2. Although the Polaris- $Q$ response times were higher than desired, they are still in a reasonable range. Unfortunately, the Ferran, TOF, MG-2100, and CDFMS exceeded the requirements by a factor of 3 or more. Based on fluid dynamics, one would expect the response times to decrease as a function of molecular mass, but no such general trend is observed.

Figure 7 displays the recovery times for each MS system. The XPR-2, UF-IT, and MG-2100 meet the requirement of $30 \mathrm{~s}$. Response times for the Polaris- $\mathrm{Q}$ and TOF exceeded the specifications by a factor of 2 , which demonstrates a strong need for improvement. The Ferran yielded response times in excess of $3 \mathrm{~min}$, which is entirely unacceptable. A few of the analyzed components exceeded the specifications for the SRS and CDFMS, but in general, these systems were deemed adequate. Again, one would have predicted lower re-

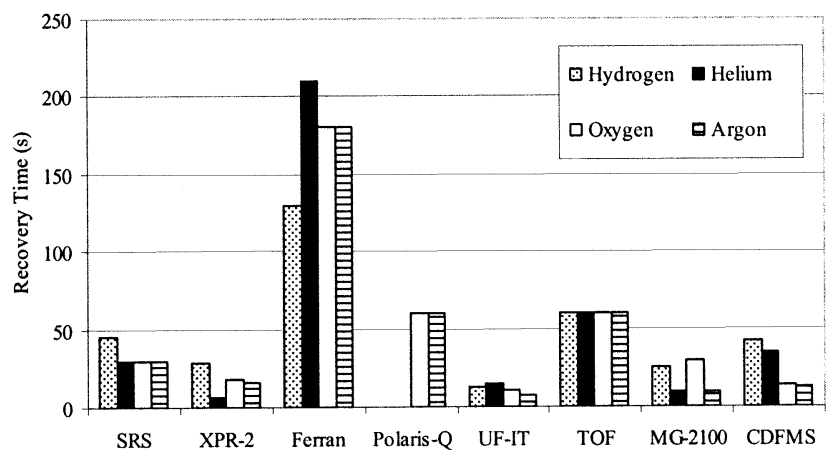

Figure 7. Comparison of the recovery times of each instrument for each species. The recovery time is measured as the time between when the test-to-zero bottle change occurs until the measured concentration is $5 \%$ of the average measured concentration of the test gas. An acceptable recovery time is $30 \mathrm{~s}$ or less. 


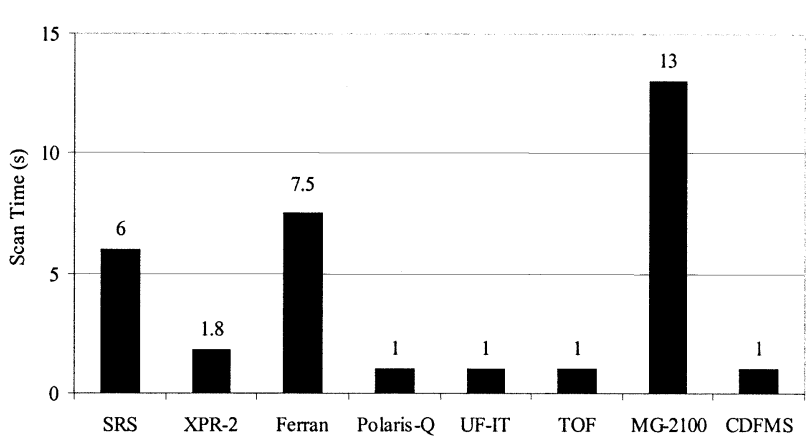

Figure 8. Comparison of the scan times for each instrument. Scan time is the time required to measure all four gases of interest in a selected ion-monitoring mode. An acceptable scan time is $2 \mathrm{~s}$ or less.

covery times for heavier components, but no such trend was observed.

One of the most important parameters that affects response and recovery time is dead volume prior to sample introduction. For the systems built in-house, great care was taken to reduce any unnecessary dead volume by placing the sample inlet as close to the SDS as possible. Also, appropriate diameter and length tubing was used to reduce dead volume while minimizing unwanted pressure reduction. Another parameter affecting response and recovery time is the flow rate of the sample gas past the sample inlet-a higher flow rate is generally better. The flow rate of the sample past the sample inlet varied from system to system. Typical values were approximately 10 to $50 \mathrm{sccm}$. Although the actual flow rate varied at the point of sample introduction, a constant flow rate $(350 \mathrm{sccm})$ was maintained at the SDS for all systems evaluated.

Figure 8 illustrates the scan time for each of the systems under study. Most of the systems have a scan time less than the 2-s requirement. The SRS, Ferran, and MG-2100 significantly exceed the requirements. The scan times for the SRS and Ferran can be reduced, but this is very detrimental to the limits of detection and other parameters.

The volume for each system is shown in Figure 9. For reference purposes, an Alcatel ATH $30+$ turbo pump has a volume of $1200 \mathrm{~cm}^{3}$. The most space-efficient systems, the XPR-2 and the CDFMS, are those that can operate using smaller backing pumps. The Ferran represents an acceptable volume. The largest system is the Polaris-Q. The primary bulk of the Polaris-Q arises from the electronics and rough pump; however, one should note that this system was designed to be a bench-top system. The second least space-efficient system, the TOF, actually has a small mass analyzer, but the electronics account for the bulk. The SRS volume is too high, primarily due to the required rough pump and the length of the quadrupole rods. The UF-IT volume is inefficient primarily as a result of its in-house construction status. It is interesting to note that the smallest system was one built in-house, even though three of the commercial systems (Ferran, TOF, and MG-2100) are

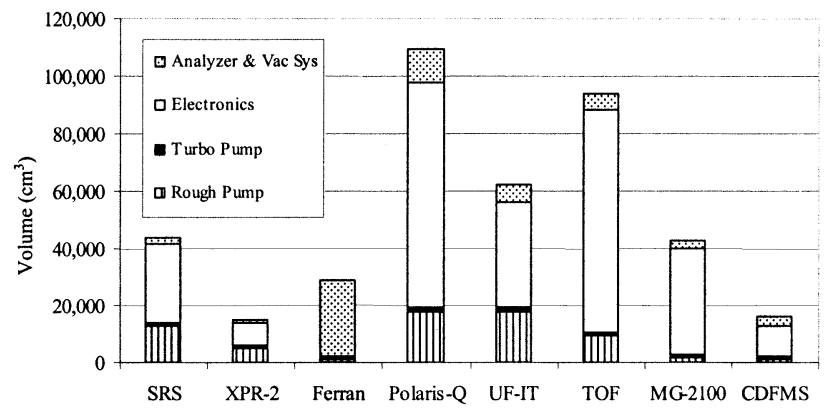

Figure 9. Comparison of the volumes of each system. The volumes of the systems, less the controlling computer and the sample delivery system, are illustrated and categorized into four subsystems: the analyzer and vacuum system, the electronics, the turbo pump, and the rough pump. A volume of $35,000 \mathrm{~cm}^{3}$ or less is acceptable. (Since the Ferran is an integrated system, it is difficult to separate the volume used by the electronics from that of other components. Since electronics are generally placed in the remaining open spaces allowed by the pumps and chamber, the volumes of the electronics and vacuum system were considered the same.)

specifically marketed, or will be marketed, as miniature MS systems.

In viewing the literature, one would think that analyzer size and performance are the major issues of miniaturization. Analyzer technology is sufficiently mature and the size is small enough that these are secondary issues. But, as can be seen in Figure 9, the primary concerns in reducing the size of the systems are the pump size [19] and the electronics. (An observation was also made by Badman and Cooks [4].)

Large portions of these systems-the vacuum chamber, the rough pump, the turbo pump, and typically the frame-are generally constructed of some form of steel. As a result, system weight is one of the more challenging requirements to meet. Only one of the systems evaluated (CDFMS) met the required 10-kg (22-lb) limit. Figure 10 compares the overall weights of each of the systems and shows a breakdown of the subsystems. Similar to the volume analysis, the rough pump and electronics account for the majority of the weight of each system.

Although not quantifiable as specific parameters, there are other aspects of the systems that should be mentioned. The Ferran and MG-2100 have undesirable sample inlet systems. The Ferran has a direct inlet system, using a capillary for pressure reduction. Although this method does provide the needed pressure reduction for the MS, it has the tendency to increase the response and recovery times. The MG-2100 also uses a capillary inlet for pressure reduction, although this system is not a direct inlet system. Instead, the MG-2100 has a flow-by stage to reduce the pressure while maintaining an acceptable flow rate. (The term "flow-by" is used to describe a sampling technique in which the sampling entrance is orthogonal to the primary gas flow.) Two problems are associated with the MG-2100 inlet design. First, the capillary protrudes beyond the 


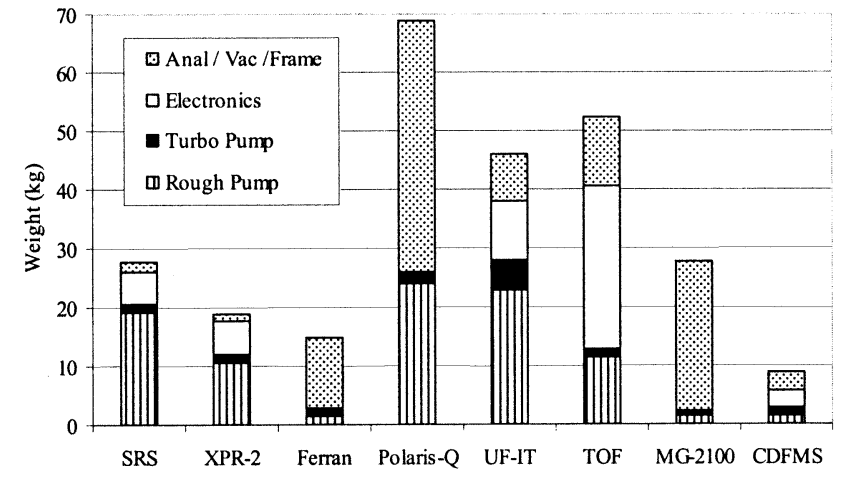

Figure 10. Comparison of the weights of each system. The weights of the systems, less the controlling computer and the sample delivery system, are illustrated and categorized into four subsystems: the analyzer/vacuum system/frame, the electronics, the turbo pump, and the rough pump. A weight of less than $10 \mathrm{~kg}$ is desired. (Since the Polaris-Q, Ferran, and MG-2100 are integrated products, it is difficult to separate the weight of the electronics from that of the other components. As a result, the weights of the electronics and vacuum system were considered the same.)

fitting, allowing the capillary to be broken on occasion. Second, even though the flow-by technique is superior for response and recovery times, the use of a capillary still deteriorates these times, relative to the use of an orifice. The Ferran had significant communication problems. This system was designed to be RS-232-compatible, yet communication errors would occur much too often (up to $50 \%$ downtime as a result). This represents a serious reliability concern. The TOF had problems with its electronics. On at least two occasions within six months, an unregulated power supply failed. The Polaris- $Q$ is unable to analyze hydrogen and helium, which is vital for the application. Although this was known from the beginning of the study, the evaluation of the Polaris-Q assisted in the design of the UF-IT and demonstrated the capabilities of a commercial ion trap. Considering applications other than that discussed here, the ion trap is of great interest due to its inherent ion manipulation and $\mathrm{MS}^{\mathrm{n}}$ capabilities [20].

Considering all of the factors involved, it is difficult to determine which system is best suited for this application. Hence, a somewhat arbitrary method was devised. As shown in Table 3, each system was given a ranking number between 1 and 10 for each of the evaluation parameters. With 1 being excellent, 5 being just within specification, and 10 being unacceptable, the categories were averaged to yield an overall score for each system. The systems considered acceptable (a score of 5 or lower) are the SRS, XPR-2, UF-IT, and CDFMS. Ideally, one or more of these four systems would have scores in the 2-to-3 range, indicating that the instrument performs well in most, if not all, categories. Scores in the 4 -to- 5 range suggest that although these systems are close to meeting the requirements, there is still much improvement needed. In order to determine which parameters need improvement, an average for each criterion was taken (the last row in Table 3). According to the average, only the scan rate is reasonable. The parameters that need the most attention appear to be the response time and system weight, with averages of 7.1 and 7.0 respectively. Although evaluating the instruments as shown in Table 3 is somewhat arbitrary, it at least provides a guide as to which instruments are worth spending time and money to improve and which parameters need the most attention.

\section{Conclusions}

A cross section of small mass spectrometer systems was evaluated, with analyzers such as linear quadrupole, quadrupole ion trap, time of flight, and sector. These systems were evaluated for the purpose of developing miniature systems for gas detection around the Space Shuttle. Many parameters were evaluated including the measurement accuracy and precision, limit of detection, response and recovery times, scan times, system volume, and system weight. The systems that were determined to work best include a linear quadrupole from Stanford Research Systems (SRS RGA-100), a miniature linear quadrupole from Inficon (XPR-2), an in-housebuilt quadrupole ion trap, and an in-house-built double-focusing sector. No system met all the requirements set forth, but, with research and modifications to these instruments, it is believed that a miniature MS system that is capable of rapid, reliable, accurate, and precise analysis is close at hand.

Future work will be focused in several areas. Improvements in limits of detection, accuracy, and preci-

Table 3. Total Ranking of Evaluated Systems

\begin{tabular}{lccccccccc}
\hline & Accuracy & Precision & LOD & Response & Recovery & Scan Rate & $\begin{array}{c}\text { System } \\
\text { Volume }\end{array}$ & $\begin{array}{c}\text { System } \\
\text { Weight }\end{array}$ & Score \\
\hline \hline SRS & 2 & 2 & 1 & 2 & 6 & 8 & 6 & $\mathbf{7}$ & $\mathbf{4 . 3}$ \\
XPR-2 & 3 & 8 & 2 & 7 & 5 & 5 & 2 & 6 & $\mathbf{4 . 8}$ \\
Ferran & 10 & 10 & 7 & 10 & 10 & 9 & 3 & 6 & $\mathbf{8 . 1}$ \\
Polaris-O & 7 & 3 & 2 & 8 & 8 & 2 & 10 & 10 & $\mathbf{6 . 3}$ \\
UF-IT & 3 & 3 & 6 & 3 & 3 & 2 & 7 & 8 & $\mathbf{4 . 4}$ \\
TOF & 9 & 6 & 7 & 9 & 7 & 2 & 9 & $\mathbf{7 . 1}$ \\
MG-2100 & 9 & 7 & 10 & 10 & 5 & 10 & 6 & $\mathbf{7}$ & $\mathbf{8 . 0}$ \\
CDFMS & 4 & 5 & 9 & 8 & 6 & 2 & 2 & 4 & $\mathbf{5 . 0}$ \\
Average & $\mathbf{5 . 9}$ & $\mathbf{5 . 5}$ & $\mathbf{5 . 5}$ & $\mathbf{7 . 1}$ & $\mathbf{6 . 3}$ & $\mathbf{5 . 0}$ & $\mathbf{5 . 6}$ & $\mathbf{7 . 0}$ & \\
\hline
\end{tabular}


sion will be addressed through the investigation of new sample introduction techniques, ionization conditions, and ion focusing. Improvements in response and recovery times are being addressed with sample introduction and gas pumping methods. Investigation into new pump technology is being used to reduce system volume and weight. As the need for miniature MS systems grows and more researchers enter the field, the time is quickly approaching when a viable miniature system is developed.

\section{References}

1. (a) Palmer, P. T.; Limero, T. F. Mass Spectrometry in the U.S. Space Program: Past, Present and Future. J. Am. Soc. Mass Spectrom. 2001, 12, 656-675. (b) Helms, W. R. History, Design and Performance of the Space Shuttle Hazardous Gas Detection System. Space Shuttle Technical Conference, Houston, TX, 1983, NASA Conference Publication 2342, Part 1, pp 573-580. (c) Helms, W. R.; Raby, B. A. A Prototype Hazardous Gas Detection System for NASA's Space Shuttle. 26th ASMS Conference on Mass Spectrometry and Allied Topics, St. Louis, MO, 1978, 463-465.

2. Griffin, T. P.; Naylor, G. R.; Haskell, W. D.; Breznik, G. S.; Mizell, C. A. A Fully Redundant On-Line Mass Spectrometric System for the Space Shuttle Used to Monitor Cryogenic Fuel Leaks. 2nd Workshop on Harsh-Environment Mass Spectrometry, March 18-21, 2001, St. Petersburg, FL.

3. Mizell, C. A.; Griffin, T. P.; Naylor, G. R.; Haskell, W. D.; Hritz, R. J. Hazardous Gas Detection System. Research and Technology 2000/2001, NASA Technical Memorandum 210258. (also located at http://technology.ksc.nasa.gov/WWWaccess/R\&T_reports. html).

4. Badman, E. R.; Cooks, R. G. Miniature Mass Analyzers. J. Am. Soc. Mass Spectrom. 2000, 35, 659-671.

5. Arkin, C R.; Ottens, A. K.; Diaz, J. A.; Griffin, T. P.; Follistein, D.; Adams, F.; Helms, W. R. Evaluating Mass Analyzers as Candidates for Small, Inexpensive, Rugged Single Point Mass Spectrometers for Analysis of Permanent Gases. Proceedings of the 49th ASMS Conference on Mass Spectrometry and Allied Topics. Chicago, IL, 2001.
6. Boumsellek, S.; Ferran, R. J. Trade-offs in Miniature Quadrupole Designs. J. Am. Soc. Mass Spectrom. 2001, 12, 633-640.

7. Ferran, R. J. U.S. Patent. Residual Gas Sensor Utilizing a Miniature Quadrupole Array. 28 March 1995, 5,401,962.

8. Ferran, R. J. U.S. Patent. Residual Gas Sensor Utilizing a Miniature Quadrupole Array. 12 January 1999, 5,857,890.

9. Ferran, R. J.; Boumsellek, S. High-pressure Effects in Miniature Arrays of Quadrupole Analyzers for Residual Gas Analysis from $10^{-9}$ to $10^{-2}$ Torr. J. Vac. Sci. Technol. A 1996, 14, 1258-1265.

10. Gonin, M; Fuhrer, K; Schultz, A. A New Concept to Increase Dynamic Range Using Multi-Anode Detectors . Proceedings of the 48th ASMS Conference on Mass Spectrometry and Allied Topics. Long Beach, CA, 2000; pp 326-327.

11. Ottens, A. K.; Griffin, T. P.; Yost, R. A. A Quadrupole Ion Trap Mass Spectrometer for Quantitative Analysis of NitrogenPurged Compartments within the Space Shuttle. 49th ASMS Conference on Mass Spectrometry and Allied Topics. : Chicago, IL, 2001.

12. Ottens, A. K.; Harrison, W. W.; Griffin, T. P.; Helms, W. R. Real-Time Quantitative Analysis of $\mathrm{H}_{2}, \mathrm{He}, \mathrm{O}_{2}$, and $\mathrm{Ar}$ by Quadrupole Ion Trap Mass Spectrometry. J. Am. Soc. Mass Spectrom. 2002, submitted.

13. Diaz, J. A.; Giese, C. F.; Gentry, W. R. Sub-Miniature ExB Sector-Field Mass Spectrometer. J. Am. Soc. Mass Spectrom. 2001, 12, 619-632.

14. Diaz, J. A. Design, Modeling and Process of a Micro-fabricated Mass Spectrometer on a Chip for Analytical Applications. MSEE Thesis. University of Minnesota, 1998.

15. Diaz, J. A. Sub-miniature Double-focusing Mass Spectrometer for In-situ Environmental Monitoring and Application to Volcanic Gaseous Emissions. Ph.D. Thesis. University of Minnesota, 1999.

14. Diaz, J. A.; Gentry, W. R.; Giese, C. F. Patent Application, University of Minnesota, 96035, 1999.

17. Griffin, T. P. Advanced Hazardous Gas Detection System (AHGDS) (Design Decisions), Requirements Document. KSCMM-4864, 10 Jan. 2001.

18. Dawson, P. H. Quadrupole Mass Spectrometry and its Applications. Elsevier: Amsterdam, 1976, pp 117-119.

19. 2nd NASA/JPL Miniature Vacuum Pumps Workshop, Pasadena, CA, 28 March 2002.

20. March, R. E. An Introduction to Quadrupole Ion Trap Mass Spectrometry. J. Mass Spectrom. 1997, 32, 351-369. 